\title{
New strategies for haploidentical transplantation
}

\author{
Lena Oevermann ${ }^{1}$ and Rupert Handgretinger ${ }^{1}$
}

Haploidentical transplantation in children opens the possibility to offer this treatment to every child with an otherwise incurable disease, such as some hematological or oncological malignancies, inborn or acquired bone marrow-failure syndromes, hemoglobinopathies, immunodeficiencies, or other genetic diseases. Although initial attempts at haploidentical transplantation were associated with a high transplant-related mortality, recent insights into the biology of haploidentical transplantation, the availability of effective in vivo large-scale graft-manipulation technology, and improved supportive care strategies have led to and are still leading to significantly better outcomes of haploidentical transplantation as compared with previous decades. In addition, expensive and time-consuming searches for matched unrelated donors (MUDs) as well as the expensive establishment and maintenance of cord blood banks are not necessary. Moreover, the worldwide donor registries comprise mainly donors of Caucasian origin and patients of non-Caucasian origin have a lower chance of finding a MUD. Therefore, haploidentical transplantation allows the treatment of children independently of their ethnic background in a timely fashion according to the status of their underlying disease.

A llogeneic hematopoietic stem cell transplantation (HSCT) is, for a number of children with malignant and nonmalignant diseases, currently the only available curative approach. The malignant diseases treated by HSCT comprise mainly hematological malignancies, whereas the nonmalignant disorders that strike mainly pediatric patients include hemoglobinopathies and inborn disorders of the immune system and metabolism. The first pediatric HSCT was performed in 1969 in a child suffering from immunodeficiency with a matched sibling donor (1). A few years later, HSCT was being used successfully to cure otherwise incurable leukemias in adults (2), and transplantations from matched sibling and matched unrelated donors (MUDs) are regarded as the current standard treatment.

As only approximately $30 \%$ of the patients requiring transplantation have a human leukocyte antigen (HLA)-matched related sibling, alternative donors such as MUDs, unrelated matched or mismatched cord blood units, or three-loci mismatched haploidentical family donors are increasingly used as sources of hematopoietic stem cells. The probability of identifying a MUD in the worldwide donor registries is dependent on the diversity of HLA antigens within a population and on the patient's race (3). In addition, a MUD donor search is time-consuming, and the time from initiation of a donor search to the actual transplant, in most cases, is 4 mo or longer (4). A significant number of patients will experience disease progression or even die within the time of donor search, and the potential life-saving allogeneic transplantation will no longer be a therapeutic option.

An alternative approach for patients without HLA-matched donors or for patients who are at high risk of disease progression during the donor search is the use of mismatched related family donors. In pediatric patients, these are mainly threeloci-mismatched parental donors, but haploidentical relatives like aunts or uncles can also be included in the rare cases when no parental donor is available or the parent is medically unfit for donation.

\section{ADVANTAGES OF HAPLOIDENTICAL TRANSPLANTATION}

Due to the immediate and permanent availability of the donor, a number of advantages as compared with other donor sources can be identified:

- A pediatric patient is diagnosed with a disease that might only be cured by HSCT and is brought to the hospital by his potential donors. There is no treatment delay and a life-saving transplant can immediately be offered.

- Based on the motivation of the donor, more recently introduced reagents such as the CXCR4 inhibitor (receptor of stromal cell-derived factor 1) for stem cell mobilization can systematically be investigated as to whether they would positively influence the outcome of transplantation (5).

- Sufficient numbers of peripheral blood stem cells (PBSCs) can be collected to allow graft-manipulation procedures to deplete $\mathrm{T}$ lymphocytes from the graft for the prevention of graft vs. host disease (GvHD). These include CD34 ${ }^{+}$positive selection (6), CD3 depletion (7), CD3/ CD19 depletion (8), and TcRaß/CD19 depletion (9).

- Bone marrow (BM) and/or peripheral stem cells can be collected, depending on the preference of the transplant center. 
- There is a choice of donor selection according to the donor's killer cell immunoglobulin-like receptor (KIR) phenotype or donor KIR haplotype, especially for adult patients with acute myeloid leukemia (10) and children with acute lymphoblastic leukemia (ALL) (11).

- The HLA disparity between donor and recipient allows a rapid and extensive analysis of the chimerism status of the recovering hematopoiesis and especially of the various lymphocyte subsets post-transplant using flow cytometry and only small amounts of blood (1 cc of blood is sufficient to extensively analyze the chimerism status of various lymphocyte and other cell subsets). In addition, the HLA disparity can also be helpful for the very early detection of impending recurrence of leukemia by flow cytometry (12).

- In the case of mixed chimerism, impending relapses or refractory viral diseases, or to accelerate immune recovery, post-transplant donor-derived adoptive therapeutic strategies can rapidly be initiated, including the adoptive transfer of purified donor-derived NK (natural killer) cells (13), adoptive transfer of T lymphocytes or T-lymphocyte subsets (14), minor histocompatibility antigen (mHA)specific T lymphocytes (15), leukemia-specific T lymphocytes (16), adoptive transfer of $\mathrm{CD} 4^{+} / \mathrm{CD} 25^{+}$regulatory $\mathrm{T}$ lymphocytes (17), adoptive transfer of virus-specific $\mathrm{T}$ cells directed against adenovirus (18), cytomegalovirus (19), Epstein-Barr virus (EBV) (20), or other donorderived effector cells to be identified in future research.

A major advantage of haploidentical donors over umbilical cord blood, a stem cell source that is also immediately available, is the availability of the donor for post-transplant cellular therapies either for prevention of relapse or the treatment of viral infections by adoptive T-cell transfer. In contrast to umbilical cord blood, where the number of stem cells is limited but important for successful transplantation (21), large numbers of haploidentical stem cells can be collected from the donor by repeated collections if necessary and these large stem cell numbers allow engraftment across the HLA-barrier (6) even after reduced toxicity conditioning regimens (22). In contrast to HLA-mismatched cord blood transplants where the transplant-related mortality (TRM) is high (23), this approach is associated with a low TRM and an acceptable rate of rejections (22). Haploidentical transplantation can therefore be seen as a platform, as illustrated in Figure 1, for the further evaluation of therapeutic strategies.

In this review, we discuss current and new strategies that will lead to further improvements and better outcome of haploidentical transplantation in children for whom this approach is the only curative treatment.

\section{MOBILIZATION OF PERIPHERAL STEM CELLS}

Historically, bone marrow was used as stem cell source in allogeneic transplantation. Subsequently, the first clinical evidence was obtained that circulating hematopoietic stem cells can completely and permanently regenerate lymphohematopoiesis after myeloablative therapy. The discovery of granulocyte colony-stimulating factor (G-CSF), granulocyte/macrophage colony-stimulating factor, and the finding that these growth factors can expand the circulating pool of stem cells led to their use to mobilize stem cells in healthy donors, including matched sibling, matched unrelated, and haploidentical donors (24). Currently, G-CSF is mainly used for stem cell mobilization in haploidentical transplantation. Moreover, the use of growth factors and the ability to mobilize and collect large numbers of stem cells have enabled clinicians to translate the concept of the "megadose approach," i.e., the transplantation of large numbers of purified haploidentical stem cells to overcome the HLA barrier (25), into clinical practice (26).

The interaction between the chemokine stromal cell-derived factor 1 and its receptor, CXCR4, in BM plays a major role in the trafficking of progenitor cells between BM and peripheral blood (27). Plerixafor is a small-molecule antagonist and reversibly inhibits the interaction of stromal cell-derived factor 1 with its cognate receptor, CXCR4, thus leading to a transient increase of the peripheral progenitor pool. A synergistic effect of plerixafor and G-CSF on mobilization of peripheral CD $34^{+}$ progenitors has been shown in healthy donors (28). It can be anticipated that the combination of plerixafor with G-CSF or granulocyte/macrophage colony-stimulating factor will be used in future clinical trials in haploidentical transplantation to further increase the number of transplanted $\mathrm{CD} 34^{+}$stem cells.

\section{GRAFT-MANIPULATION STRATEGIES}

A number of initial attempts using haploidentical nonT-cell-depleted (T-cell replete) unmanipulated bone marrow and myeloablative conditioning have been made in the past.

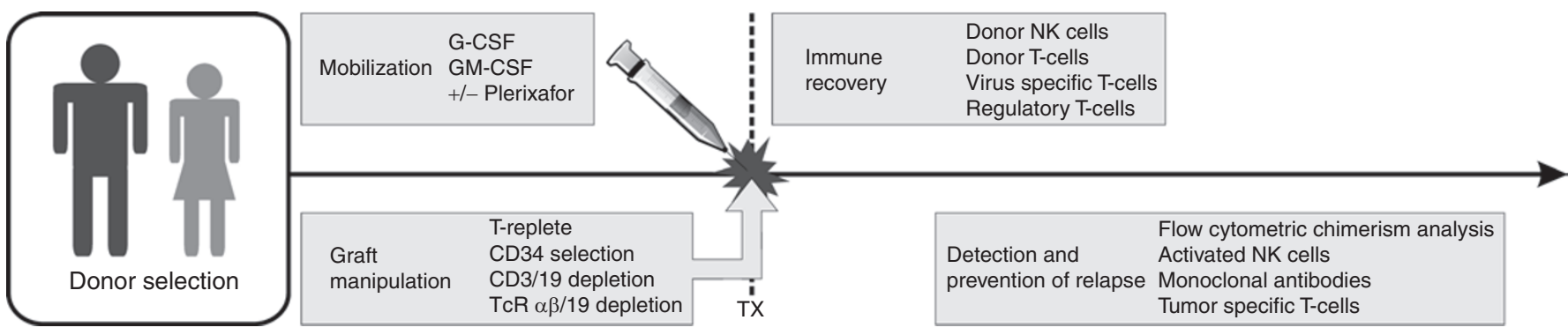

Figure 1. Haploidentical transplantation is a platform for optimizing allogeneic cellular immunotherapy. Starting from new donor-selection criteria, new methods of stem cell mobilization, graft-processing strategies, posttransplant adoptive transfer of donor-derived effector cells, and new strategies to detect and prevent relapses can systematically be evaluated. 
Unfortunately, these attempts were associated with early severe and often lethal side effects, including pulmonary edema and multiorgan failure resembling hyperacute GvHD (29). The small number of patients receiving transplants from an HLA 2- or 3-antigen mismatched related bone marrow donor experienced high and early TRM. Based on these previous findings, 2- or 3-antigen mismatched transplantation of T-cell replete bone marrow from a family donor using pharmacological GvHD prophylaxis was considered too risky.

\section{PREVIOUS EXPERIENCE WITH POORLY T-CELL-DEPLETED BONE MARROW}

As donor-derived $\mathrm{T}$ lymphocytes in the graft are the major cause of GvHD in allogeneic HSCT, various attempts were made to deplete $\mathrm{T}$ cells from BM prior to infusion. The first ex vivo T-cell depleted bone marrow transplants using soybean agglutinin and rosette formation with sheep red blood cells were performed in children with immunodeficiency syndromes (30). Subsequently, Henslee-Downey and colleagues used intensive total body irradiation (TBI)-based myeloablative preparative regimens with partial in vitro T-cell depletion of haploidentical BM using anti-CD3 antibodies (T10B9 or OKT-3) with a 1-1.5 log reduction of T cells and posttransplant pharmacological GvHD prophylaxis (31). In this large series of 201 patients, 98\% engrafted with 5-year cumulative incidences of relapse and TRM of $31 \%$ and $51 \%$, respectively.

\section{EXPERIENCE WITH HIGHLY PURIFIED CD34+ MOBILIZED PERIPHERAL STEM CELLS}

To decrease the risk of GvHD by a higher degree of ex vivo T-cell depletion, more efficient T-cell depletion technologies were introduced (32), and the possibility of using highly purified $\mathrm{CD}_{34}{ }^{+}$stem cells from mobilized peripheral blood from parental donors led investigators to evaluate haploidentical transplantation using large numbers of highly purified CD $34^{+}$ stem cells in patients with a variety of diseases. With this extensive T-cell depletion, almost no GvHD was seen even in the absence of any pharmacological GvHD prophylaxis, and due to the indirect B-cell depletion associated with $\mathrm{CD}_{3}{ }^{+}$selection, post-transplant Epstein-Barr virus-related lymphoproliferative disorders were also absent in both pediatric $(6,33)$ and adult patients (34). However, the experience of the transplant center with haploidentical transplantation of CD34 ${ }^{+}$stem cells had a major impact on the outcome. In an analysis on behalf of the Acute Leukemia and Pediatric Disease Working Parties of the European Group for Blood and Marrow Transplant, on the outcome of 102 children with ALL in remission receiving haploidentical transplants with $\mathrm{CD} 34^{+}$positively selected stem cells, impacts of the number of transplanted CD34 ${ }^{+}$stem cells and the transplant center size (in terms of number of haploidentical transplants performed per year) were demonstrated (35). All these studies in both pediatric and adult patients had a delayed immune reconstitution in common, which was associated with a high incidence of severe and lethal infections. To accelerate the post-transplant immune reconstitution, negative depletion strategies of T- and B-cells were evaluated.

\section{EXPERIENCE WITH T- AND B-CELL-DEPLETED STEM CELLS}

There is a fundamental difference between positive selection of CD34+ stem cells and the negative depletion of unwanted cells. With positively selected grafts, no other cells are co-transplanted whereas negatively depleted grafts retain, besides the $\mathrm{CD} 4^{+}$stem cells, large numbers of other cells including NK cells, monocytes, dendritic cells, and other mobilized myeloid cells. Both methods result in an effective depletion of T- and B-cells.

\section{Depletion of $\mathrm{CD}^{+} / \mathrm{CD} 19^{+}$Lymphocytes}

The introduction of semiautomated devices for the concomitant depletion of T- and B-lymphocytes to prevent GvHD and post-transplant Epstein-Barrvirus-associated lymphoproliferative disease, respectively, allows the effective depletion of $\mathrm{CD}^{+}$ and $\mathrm{CD} 19^{+}$lymphocytes (CD3/19 depletion) from mobilized peripheral stem cell grafts (8). The T-cell depletion with this method (3.5-4 log) is less as compared with the $\mathrm{CD} 34^{+}$positive selection method $(4.5-5 \mathrm{log})$. In contrast to the $\mathrm{CD} 34^{+}$positive selection method, large numbers of NK cells, monocytes, dendritic cells, and other myeloid cells are retained in the graft. In the first clinical study, 20 children with hematological malignancies were transplanted with CD3-depleted PBSCs following a TBI-based myeloablative preparative regimen (36). Of 19 patients evaluable for engraftment, all engrafted. Six patients (30\%) died from TRM and 4 patients (20\%) from disease recurrence. Ten patients (50\%) were alive and well. A subsequent pediatric study was performed in 25 patients with advanced refractory hematological malignancies $(n=9)$ or patients with relapse after previous standard myeloablative transplantation $(n=16)$ (37). As these patients were considered to be at high risk of TRM with a myeloablative preparative regimen, a less intensive non-TBI-based conditioning regimen consisting of fludarabine $\left(200 \mathrm{mg} / \mathrm{m}^{2}\right)$, thiotepa $(10 \mathrm{mg} / \mathrm{kg})$, melphalan $\left(120 \mathrm{mg} / \mathrm{m}^{2}\right)$, and the anti-CD3 antibody OKT-3 was employed. Two of 3 patients who did not engraft were rescued by another transplant from their original donor, and 1 patient had early disease progression without donor engraftment. The cumulative incidences of grade II-IV and grade III-IV GvHD were $44 \%$ and $8 \%$, respectively. The cumulative incidence of chronic GvHD was $28 \%$. Thirteen patients died of disease recurrence and 4 of TRM. No lethal viral infections were seen. Eight patients remain alive with a performance score greater than or equal to $90 \%$.

In a comparative analysis of the immune reconstitution in these two cohorts of patients, a much faster recovery of thymopoiesis, as determined by rapid increase of T-cell receptor excision circles, and a rapid return of the T-cell receptor repertoire were seen in patients who received a non-TBI-based less intensive preparative regimen as compared with the patients who received myeloablative TBI (38).

Based on these promising results, additional studies were then initiated in pediatric and adult patients using the less intensive non-TBI-based preparative regimen, consisting of fludarabine $\left(160 \mathrm{mg} / \mathrm{m}^{2}\right)$, thiotepa $(10 \mathrm{mg} / \mathrm{kg})$, melphalan $\left(140 \mathrm{mg} / \mathrm{m}^{2}\right)$, and OKT-3 (22). In a study of 38 pediatric patients, primary 
engraftment was seen in $83 \%$. The major cause of graft failure was rejection. However, after reconditioning and a second transplant with CD3/19-depleted PBSCs from the other haploidentical parental donor (39), engraftment was finally obtained in $98 \%$ of the patients. Most importantly, only 1 of 38 patients died from TRM. Acute GvHD grades $0-I$, II, and III-IV was seen in $73 \%, 24 \%$, and $3 \%$ of the patients, respectively. In a recent update, Pfeiffer et al. reported an event-free survival of $51 \%$ for children with acute leukemia who were in morphological remission at the time of transplantation (40). A similar promising outcome was recently reported in a pediatric study of haploidentical transplantation using CD3/19-depleted PBSCs from Uruguay (41). It is noteworthy that in this study 3 patients with Fanconi anemia were successfully transplanted from haploidentical donors.

\section{Depletion of $\mathrm{TcRa} \beta \pm / C D 19 \pm$ Lymphocytes}

As compared with $\mathrm{CD} 34^{+}$positive selection, the number of graft-contaminating $\mathrm{T}$ cells is approximately tenfold higher in CD3/19-depleted grafts, requiring short-term post-transplant immunosuppression with mycophenolate mofetil (22). A more effective approach to negative depletion of $\mathrm{T}$ cells is the recently described negative depletion of T-cell receptor (TcR) $\alpha \beta^{+} T$ lymphocytes from mobilized peripheral stem cell grafts (9). With this method, a T-cell reduction of 4.5-5 log can be achieved, which is comparable to $\mathrm{CD} 34^{+}$positive selection. This approach retains NK cells, monocytes, dendritic cells, and $\gamma \delta^{+}$ T lymphocytes in the graft. $\gamma \delta^{+}$T lymphocytes are nonalloreactive lymphocytes with important anti-infectious and antitumor properties $(42,43)$ that might have an impact on the outcome of haploidentical transplantation. In this context, it has been reported that $\gamma \delta^{+} \mathrm{T}$ cells can exert an antileukemic effect in partially mismatched HSCT (44). In this retrospective analysis, patients with a high $\gamma \delta$ T-cell count posttransplant had a better 5 -year survival than those with normal or low counts (70.8 vs. $19.6 \%, P=0.0001$ ), whereas no difference in the incidence of GvHD was observed. The first clinical experiences in children undergoing haploidentical transplantation of TcR $\alpha \beta / C D 19-$ depleted T cells obtained at the Children's University Hospital Tuebingen are promising, with a rapid donor engraftment, a rapid early expansion of donor-derived $\gamma \delta^{+} \mathrm{T}$ lymphocytes, and a rapid immune reconstitution with a median time to reach 100 $\mathrm{CD}^{+} \mathrm{T}$ cells of 34 days (own unpublished observations).

\section{CURRENT EXPERIENCE WITH T-CELL-REPLETE BONE MARROW AND/OR PERIPHERAL STEM CELLS}

More recently, T-cell-replete bone marrow with or without PBSCs has been reintroduced in combination with intensive pharmacological prophylaxis for GvHD or with the in vivo T-cell depletion of donor-derived $\mathrm{T}$ cells after infusion of the graft using polyclonal antithymocyte globulin or monoclonal antibodies (alemtuzumab). Huang et al. combined G-CSF-primed $\mathrm{BM}$ and unmanipulated PBSCs following a myeloablative preparative regimen in adults (45). All patients achieved full donor chimerism. Grade II-IV acute GvHD occurred in $78 \%$ and chronic GvHD in $73 \%$ of the patients. In another large study,
HLA-mismatched or haploidentical blood and marrow transplantation achieved comparable outcomes with HLA-identical sibling transplantation (46). In this setting, the graft composition affected the clinical outcome, and a higher $\mathrm{CD} 4 / \mathrm{CD} 8$ ratio in the G-CSF-primed BM was associated with a survival disadvantage and a trend toward relapse, whereas a lower CD4/CD8 ratio in primed $\mathrm{BM}$ was associated with a survival benefit (47). The use of G-CSF-mobilized PBSCs alone was inferior as compared with the use of G-CSF-primed BM and G-CSF-mobilized PBSCs (48). G-CSF-mobilized BM alone was used in pediatric patients with hematological malignancies after myeloablative conditioning. For GvHD prophylaxis, antithymocyte globulin, basiliximab (a chimeric mouse-human monoclonal antibody that recognizes the a chain (CD25) of the IL-2 receptor of T cells), cyclosporine $\mathrm{A}$, and short-course methotrexate were used (49). The 2-year disease-free survival was 53\%.

\section{Post-Transplant High-Dose Cyclophosphamide}

A more recently described promising approach is in vivo allodepletion using high-dose cyclophosphamide (CY) posttransplant to induce tolerance. In these protocols, donor-derived lymphocytes are exposed to host antigens that induce proliferation of antigen-specific lymphocytes. It is hypothesized that by timely exposure to post-transplant CY, proliferating lymphocytes are killed whereas resting lymphocytes are spared $(50,51)$. In a study of 68 patients with hematological malignancies, CY $(50 \mathrm{mg} / \mathrm{kg})$ was given on day 3 or days 3 and 4 after nonmyeloablative conditioning and transplantation of T-cellreplete haploidentical BM (52). Graft failure occurred in 13\% of the patients and the cumulative incidences of acute grade II-IV, grade III-IV GvHD, and chronic GvHD were $34 \%, 6 \%$, and $22 \%$, respectively. Actuarial overall and event-free survival (EFS) at 2 years after transplantation were $36 \%$ and $26 \%$, respectively. More recently, promising results of a phase 2 study using partially HLA-mismatched related bone marrow grafts have been reported (53).

\section{WHO IS THE BEST HAPLOIDENTICAL DONOR?}

In the vast majority of pediatric patients, the donor will either be the patient's mother or father. Therefore, the best donor has to be selected from these two. But who is the best donor? The best donor will be the one whose graft and reconstituting lymphohematopoiesis exerts the most effective graft-versus-leukemia effect in the absence of GvHD and facilitates rapid, complete, and permanent engraftment of donor hematopoiesis.

\section{The Role of Alloreactive Natural Killer Cells}

The broader use of highly purified CD34 ${ }^{+}$stem cells in haploidentical HSCT has allowed deep insights into the biology of NK cells and into the understanding of NK alloreactivity $(54,55)$. NK cells express KIRs on their surface, which, upon binding to their cognate ligands, i.e., certain HLA alleles, induce an inhibitory or activatory signal. These signals, together with other signals, result either in the inhibition or activation of NK cells (56). An overview on inhibitory and activatory KIRs and their known ligands is summarized in Table 1 . In the setting 
Table 1. KIRs and their ligands

\begin{tabular}{ll}
\hline KIR & Ligand \\
\hline $2 D L 1$ & HLA-C2 epitope \\
$2 D L 2$ & HLA-C1 epitope \\
$2 D L 3$ & HLA-C1 epitope \\
$2 D L 4$ & HLA-G \\
$2 D L 5$ & Unknown \\
$3 D L 1$ & HLA-B and HLA-A \\
$3 D L 2$ & HLA-A3,-A11 \\
$3 D L 3$ & Unknown \\
$3 D S 1$ & Unknown \\
$2 D S 1$ & HLA-C2 epitope \\
$2 D S 2$ & Unknown \\
$2 D S 3$ & Unknown \\
$2 D S 4$ & HLA-A11, subsets of $-C$ \\
$2 D S 5$ & Unknown \\
\hline
\end{tabular}

Most of the ligands for activatory KIRs (italics) are unknown, whereas inhibitory KIRs mainly recognize HLA-C epitopes. HLA, human leukocyte antigen; KIR, killer cell immunoglobulin-like receptor.

of allogeneic and especially haploidentical transplantation, donor-derived alloreactive NK cells can lyse tumor cells if there is a mismatch between the KIR receptors and their corresponding ligands on the target cells (57). Mismatching donor KIR receptors with recipient HLA alleles (ligands) can provoke a powerful graft vs. leukemia effect, especially in the absence of GvHD (58).

Different models have been described to predict NK cell alloreactivity. There is the ligand-ligand model built on the "missing self" hypothesis, which assumes that each individual must express at least one KIR for self-HLA alleles. According to this model, an NK alloreactive situation is predicted if there is a mismatch of KIR ligands (i.e., mismatch of certain HLA alleles) between donor and recipient. In the clinical setting of haploidentical transplantation, this model was valuable for predicting a powerful antileukemic effect in adult patients with acute myeloid leukemia but not with ALL (10).

The receptor-ligand model is based on the direct determination of the donor's KIR repertoire and the patient's KIR ligands to predict NK alloreactivity $(11,59)$. Lacking the corresponding ligands, leukemic blasts cannot inhibit the donor's NK cells, and tumor lysis is initiated. And indeed, a significant reduced risk of relapse was seen in pediatric patients after KIR receptor-ligand mismatched haploidentical transplantation with $\mathrm{CD}_{3} 4^{+}$selected cells $(11,60)$. The receptor-ligand model is depicted in Figure 2.

There is less evidence about the role of donor-derived NK cell receptors and their ligands in CD3/19-depleted haploidentical transplantation. When Pfeiffer et al. investigated the NK cell receptor repertoire reconstitution in pediatric patients after CD3/19 T-depleted haploidentical transplantation using a nonmyeloablative regimen, a prevalent early expansion of $\mathrm{CD}_{158 \mathrm{~b}^{+}} \mathrm{NK}$ cells was seen. Patients
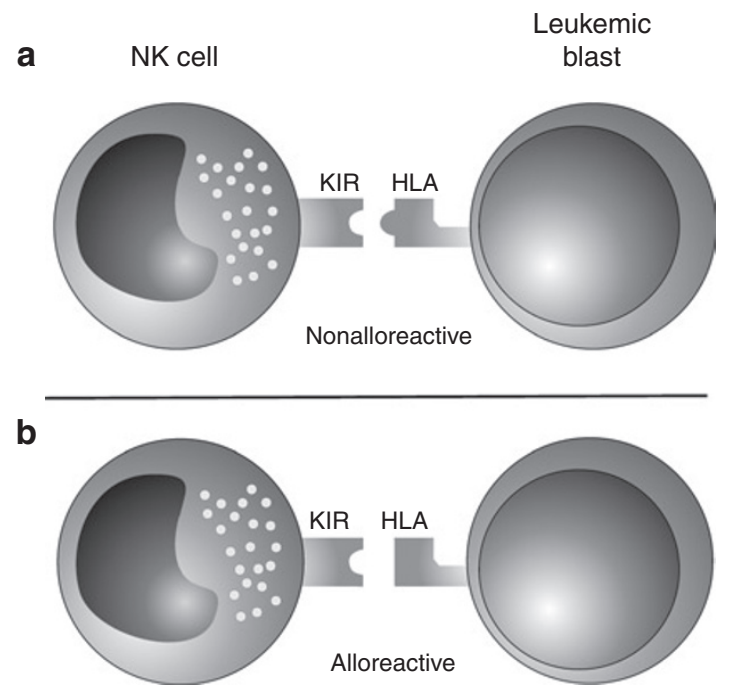

Figure 2. Receptor-ligand model: (a) If a killer cell immunoglobulin-like receptor (KIR) is engaged by its cognate HLA ligand, the NK cell function is inhibited and the donor NK cell is nonalloreactive toward the patient's blasts. (b) If the KIR is not engaged by the patient's HLA, the donor NK cell is alloreactive and can kill the patient's blasts. HLA, human leukocyte antigen; NK, natural-killer.

homozygous for the corresponding inhibitory KIR ligands (certain HLA-Cw alleles) had a poorer outcome than the ones that were heterozygous (40). Similar findings were recently seen for haploidentical transplantations with $\mathrm{CD} 34^{+}$ positively selected stem cells (61).

In T-cell-replete or poorly T-cell-depleted allogeneic transplantation, the effect of alloreactive NK cells might be overridden by alloreactive $\mathrm{T}$ cells requiring intensive pharmacologic GvHD prophylaxis (62). This could explain the deleterious effects of KIR ligand incompatibility on clinical outcomes in haploidentical hematopoietic stem cell transplantation without in vitro T-cell depletion that were seen (63). However, an important role of the donor KIR repertoire in haploidentical transplantation was reported by Symons et al. (64). They examined 86 patients with advanced hematologic malignancies who received nonmyeloablative, HLA-haploidentical HSCT with high-dose, posttransplant cyclophosphamide. Patients homozygous for an inhibiting or KIR A haplotype had an improved overall survival, event-free survival, and nonrelapse mortality if their donor had a KIR B/B or B/x haplotype, which encodes several activating KIRs.

The KIR haplotype model was further developed by Cooley et al. They analyzed the influence of the KIR genotype on the outcome in a cohort of 1,409 adult patients with either acute myeloid leukemia or ALL after matched unrelated HSCT. In patients who received a graft from a donor who had a KIR B/B haplotype, i.e., a high number of activating KIRs, a significant reduced risk of relapse was observed (65). Furthermore, the content of activating KIRs was quantified using a score from 0 to 4 , with 0 indicating a low and 4 a high number of activating KIRs, and donors with the highest haplotype B content score conferred the lowest risk of relapse. The KIR haplotype model is illustrated in Figure 3. 


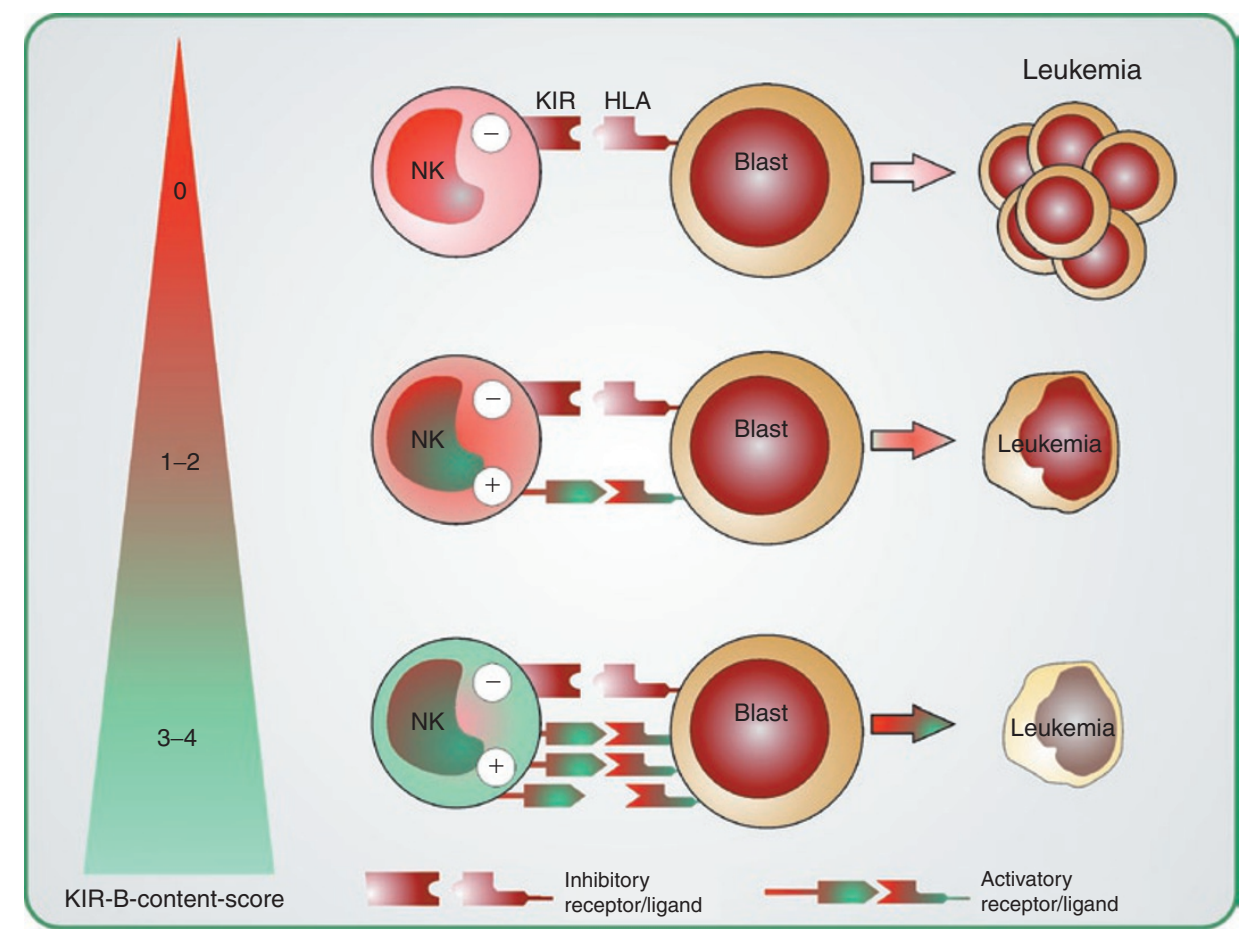

Figure 3. In the setting of haploidentical HSCT haplotype A donor NK cells, cells with only inhibitory KIRs (KIR-B-content score of 0 ) are not capable of lysing leukemic blasts, whereas haplotype B donor NK cells, which exhibit one or more activatory KIRs may induce lysis of leukemic blasts in the recipient. Therefore, the antileukemic effect is growing with an increasing KIR-B-content score that is equivalent to the number of activatory KIRs on the donor NK cells. HSCT, hematopoietic stem cell transplantation; KIR, killer cell immunoglobulin-like receptor; NK, natural-killer.

In pediatric patients with very-high-risk ALL, a significant reduction of relapse was seen after haploidentical transplantation with $\mathrm{CD} 34^{+}$-selected or $\mathrm{CD} 3 / 19$-depleted grafts in patients transplanted from a donor exhibiting a KIR B haplotype. By applying the Cooley KIR-B-content-score, the risk of relapse was lowest for the highest KIR-B-content score (own unpublished data). These observations are in line with a more recent case-control study including 109 children suffering from ALL. Almalte et al. found that inheriting a higher number of activating KIR genes was associated with significant reduction in risk for development of ALL (66). These findings support the important influence of activating KIRs in pediatric ALL not only in the setting of haploidentical transplantation but also for the development and maybe the course and therapy of pediatric ALL.

\section{REBUILDING THE IMMUNE SYSTEM AFTER HAPLO- IDENTICAL TRANSPLANTATION}

A delayed reconstitution of the immune system in children and adults is an overall obstacle after allogeneic HSCT (67). The delayed and insufficient T-cell reconstitution observed after haploidentical HSCT causes an especially high rate of infections and consequent TRM (68). New strategies to improve the T-cell reconstitution include the adoptive transfer of donorderived virus-specific $T$ cells $(18,19)$, adoptive immunotherapy with allodepleted donor T cells (69), the use of donor T cells selectively depleted of alloreactive lymphocytes by photodepletion (70), the use of donor pathogen-specific T cells (71), or the posttransplant infusion of CD8-depleted donor lymphocytes (72).

In mouse models, Edinger et al. found that $\mathrm{CD} 4{ }^{+} \mathrm{CD} 25^{+}$ positive regulatory $\mathrm{T}$ cells preserved graft-versus-tumor activity while they were inhibiting development of GvHD (73). This finding contributes to another new approach in immune reconstitution, the adoptive transfer of regulatory $\mathrm{T}$ cells after transplantation; Di Ianni et al. could prevent GvHD, promote lymphoid reconstitution, improve immunity to opportunistic pathogens, and induce a strong graft-versus-leukemia effect after infusion of donor-derived $\mathrm{CD} 4^{+} \mathrm{CD} 25^{+}$-positive regulatory $\mathrm{T}$ cells in 28 patients after haploidentical transplantation of positively selected CD $34^{+}$stem cells followed by conventional $\mathrm{T}$ cells without any post-transplant immunosuppression (17). In addition, the immune recovery was significantly improved without development of GvHD.

The influence on the immune reconstitution of the T-cell depletion method combined with a reduced myeloablative regimen remains unclear. So far, a faster immune reconstitution in adults(74) and no lethal infections in children(22) have been observed following a nonmyeloablative regimen with $\mathrm{CD} 3 /$ CD19-depleted grafts for haploidentical HSCT. In this setting, the thymic function might play an important role. A faster T-cell reconstitution was seen in patients exhibiting a better thymic-dependent T-cell function before transplantation (75). In addition, the immune reconstitution was faster in pediatric patients transplanted following a reduced-intensity regimen with a CD3/CD19-depleted graft than in patients transplanted 
with a CD3-depleted graft containing TBI-based myeloablation (38). Future research should show whether the rapidly expanding $\gamma \delta^{+}$T lymphocytes after transplantation of TcRa $\beta /$ CD19-depleted PBSCs will have any anti-infectious protective effect.

\section{ATTEMPTS TO PREVENT RELAPSE}

The main challenge in haploidentical transplantation is to keep the balance between conserving the graft-versusleukemia effect on the one hand and preventing GvHD on the other. T cells play a crucial role in leukemia surveillance after HSCT, and donor lymphocyte infusions might contribute in some hematological malignancies (14). The important role of $\mathrm{T}$ cells for the control of leukemia was further shown by Vago et al. (76). In this study, 5 of 17 patients relapsed after HLA-mismatched HSCT. Leukemic cells were lacking HLAmismatched alleles after HSCT with consecutive total loss of the HLA-mismatched haplotype. This loss was found to be due to an acquired uniparental disomy on the short arm of chromosome 6. Mutant variants of the leukemic cells escaped T-cell dependent surveillance and caused relapse. A similar tumorescape mechanism was also found in children after haploidentical HSCT (77). However, even small amounts of donor T cells can cause GvHD without adequate immunosuppression in haploidentical transplantation (78). Solutions to circumvent GvHD development might be adoptive transfer of NK-cellenriched donor lymphocyte infusions (79) or infusions with ex vivo interleukin-15-stimulated NK cells (80).

Other strategies to prevent relapse might be the in vivo infusion of monoclonal antibodies directed against the CD19 antigen expressed in pediatric ALL after transplantation (81). More recently, a CD3/CD19 bispecific T-cell-engaging antibody (blinatumomab) was used to treat post-transplant relapses in pediatric patients with ALL (82). Three patients treated with blinatumomab showed minimal residual disease-negative remission (83). The fact that 2 of the 3 patients were treated after haploidentical transplantation and did not develop GvHD despite the T-cell-engaging antibody is particularly worth mentioning.

Besides developing new strategies to prevent relapse, precise methods of detecting early relapse are likewise highly relevant. Chimerism analysis can easily and rapidly be done using anti-HLA antibodies conjugated to fluorochromes, as host and donor cells can be differentiated due to the existent HLA-mismatch in haploidentical transplantation (12). The detection of residual host hematopoiesis can be combined with the flow cytometric detection of minimal residual disease (84) and allows very early detection of an impending relapse and an immediate intervention for its prevention.

\section{CONCLUSIONS}

Advances in haploidentical HSCT over the past years have raised significant interest in this approach. It is still an evolving field, and many further improvements can be envisioned over the next years. Due to the continuous availability of the donor, posttransplant strategies to improve immune reconstitution and to prevent disease recurrence can be designed. Given the current promising clinical results in children and adults, haploidentical transplantation should no longer be regarded as a last-resort treatment for hopeless patients but should be offered to patients with an indication for allogeneic transplant who do not have a matched sibling or a MUD identified within a reasonable time frame. Using this approach, very promising results have been reported. Children with leukemia (85) and patients with veryhigh-risk leukemia who received a haploidentical transplant using either $\mathrm{CD}_{3} 4^{+}$stem cells or $\mathrm{CD} 3 / 19$-depleted stem cells in complete remission had a 5 -year overall survival of $88 \%$.

\section{REFERENCES}

1. Gatti RA, Meuwissen HJ, Allen HD, Hong R, Good RA. Immunological reconstitution of sex-linked lymphopenic immunological deficiency. Lancet 1968;2:1366-9.

2. Thomas ED, Buckner CD, Banaji M, et al. One hundred patients with acute leukemia treated by chemotherapy, total body irradiation, and allogeneic marrow transplantation. Blood 1977;49:511-33.

3. Beatty PG, Boucher KM, Mori M, Milford EL. Probability of finding HLAmismatched related or unrelated marrow or cord blood donors. Hum Immunol 2000;61:834-40.

4. Heemskerk MB, van Walraven SM, Cornelissen JJ, et al. How to improve the search for an unrelated haematopoietic stem cell donor. Faster is better than more! Bone Marrow Transplant 2005;35:645-52.

5. Neumann T, Krüger WH, Busemann C, Kiefer T, Dölken G. Successful mobilization of PBSCs in a healthy volunteer donor by addition of plerixafor after failure of mobilization with G-CSF alone. Bone Marrow Transplant 2011;46:762-3.

6. Handgretinger R, Klingebiel T, Lang P, et al. Megadose transplantation of purified peripheral blood CD34(+) progenitor cells from HLA-mismatched parental donors in children. Bone Marrow Transplant 2001;27:777-83.

7. Gordon PR, Leimig T, Mueller I, et al. A large-scale method for T cell depletion: towards graft engineering of mobilized peripheral blood stem cells. Bone Marrow Transplant 2002;30:69-74.

8. Barfield RC, Otto M, Houston J, et al. A one-step large-scale method for T- and B-cell depletion of mobilized PBSC for allogeneic transplantation. Cytotherapy 2004;6:1-6.

9. Chaleff S, Otto M, Barfield RC, et al. A large-scale method for the selective depletion of alphabeta T lymphocytes from PBSC for allogeneic transplantation. Cytotherapy 2007;9:746-54.

10. Ruggeri L, Mancusi A, Capanni M, et al. Donor natural killer cell allorecognition of missing self in haploidentical hematopoietic transplantation for acute myeloid leukemia: challenging its predictive value. Blood 2007;110:433-40.

11. Leung W, Iyengar R, Turner V, et al. Determinants of antileukemia effects of allogeneic NK cells. J Immunol 2004;172:644-50.

12. Schumm M, Feuchtinger T, Pfeiffer M, et al. Flow cytometry with anti HLA-antibodies: a simple but highly sensitive method for monitoring chimerism and minimal residual disease after HLA-mismatched stem cell transplantation. Bone Marrow Transplant 2007;39:767-73.

13. Triplett B, Handgretinger R, Pui CH, Leung W. KIR-incompatible hematopoietic-cell transplantation for poor prognosis infant acute lymphoblastic leukemia. Blood 2006;107:1238-9.

14. Kolb HJ. Graft-versus-leukemia effects of transplantation and donor lymphocytes. Blood 2008;112:4371-83.

15. Bleakley M, Riddell SR. Exploiting $\mathrm{T}$ cells specific for human minor histocompatibility antigens for therapy of leukemia. Immunol Cell Biol 2011;89:396-407.

16. Rezvani K, Barrett AJ. Characterizing and optimizing immune responses to leukaemia antigens after allogeneic stem cell transplantation. Best Pract Res Clin Haematol 2008;21:437-53.

17. Di Ianni M, Falzetti F, Carotti A, et al. Tregs prevent GVHD and promote immune reconstitution in HLA-haploidentical transplantation. Blood 2011;117:3921-8. 
18. Feuchtinger T, Matthes-Martin S, Richard C, et al. Safe adoptive transfer of virus-specific T-cell immunity for the treatment of systemic adenovirus infection after allogeneic stem cell transplantation. Br J Haematol 2006;134:64-76.

19. Feuchtinger T, Opherk K, Bethge WA, et al. Adoptive transfer of pp65specific T cells for the treatment of chemorefractory cytomegalovirus disease or reactivation after haploidentical and matched unrelated stem cell transplantation. Blood 2010;116:4360-7.

20. Moosmann A, Bigalke I, Tischer J, et al. Effective and long-term control of EBV PTLD after transfer of peptide-selected T cells. Blood 2010;115:2960-70.

21. Gluckman E. Milestones in umbilical cord blood transplantation. Blood Rev 2011;25:255-9.

22. Handgretinger R, Chen X, Pfeiffer M, et al. Feasibility and outcome of reduced-intensity conditioning in haploidentical transplantation. Ann N Y Acad Sci 2007;1106:279-89.

23. Eapen M, Rubinstein P, Zhang MJ, et al. Outcomes of transplantation of unrelated donor umbilical cord blood and bone marrow in children with acute leukaemia: a comparison study. Lancet 2007;369:1947-54.

24. Körbling M, Freireich EJ. Twenty-five years of peripheral blood stem cell transplantation. Blood 2011;117:6411-6.

25. Rachamim N, Gan J, Segall H, et al. Tolerance induction by "megadose" hematopoietic transplants: donor-type human CD34 stem cells induce potent specific reduction of host anti-donor cytotoxic $\mathrm{T}$ lymphocyte precursors in mixed lymphocyte culture. Transplantation 1998;65: 1386-93.

26. Aversa F, Tabilio A, Velardi A, et al. Treatment of high-risk acute leukemia with T-cell-depleted stem cells from related donors with one fully mismatched HLA haplotype. N Engl J Med 1998;339:1186-93.

27. Petit I, Szyper-Kravitz M, Nagler A, et al. G-CSF induces stem cell mobilization by decreasing bone marrow SDF-1 and up-regulating CXCR4. Nat Immunol 2002;3:687-94.

28. Liles WC, Broxmeyer HE, Rodger E, et al. Mobilization of hematopoietic progenitor cells in healthy volunteers by AMD3100, a CXCR4 antagonist. Blood 2003;102:2728-30.

29. Powles RL, Morgenstern GR, Kay HE, et al. Mismatched family donors for bone-marrow transplantation as treatment for acute leukaemia. Lancet $1983 ; 1: 612-5$.

30. Friedrich W, Goldmann SF, Vetter U, et al. Immunoreconstitution in severe combined immunodeficiency after transplantation of HLA-haploidentical, T-cell-depleted bone marrow. Lancet 1984;1:761-4.

31. Mehta J, Singhal S, Gee AP, et al. Bone marrow transplantation from partially HLA-mismatched family donors for acute leukemia: single-center experience of 201 patients. Bone Marrow Transplant 2004;33:389-96.

32. Schumm M, Lang P, Taylor G, et al. Isolation of highly purified autologous and allogeneic peripheral CD34+ cells using the CliniMACS device. J Hematother 1999;8:209-18.

33. Peters C, Matthes-Martin S, Fritsch G, et al. Transplantation of highly purified peripheral blood CD34+ cells from HLA-mismatched parental donors in 14 children: evaluation of early monitoring of engraftment. Leukemia 1999;13:2070-8.

34. Aversa F, Terenzi A, Tabilio A, et al. Full haplotype-mismatched hematopoietic stem-cell transplantation: a phase II study in patients with acute leukemia at high risk of relapse. J Clin Oncol 2005;23:3447-54.

35. Klingebiel T, Cornish J, Labopin M, et al.; Pediatric Diseases and Acute Leukemia Working Parties of the European Group for Blood and Marrow Transplantation (EBMT). Results and factors influencing outcome after fully haploidentical hematopoietic stem cell transplantation in children with very high-risk acute lymphoblastic leukemia: impact of center size: an analysis on behalf of the Acute Leukemia and Pediatric Disease Working Parties of the European Blood and Marrow Transplant group. Blood 2010;115:3437-46.

36. Hale GA, Kasow K, Gan K, et al. Haploidentical Stem Cell Transplantation with CD3 Depleted Mobilized Peripheral Blood Stem Cell Grafts for Children with Hematologic Malignancies. 47th Annual Meeting of the American Society of Hematology, 10-13 December 2005, Atlanta, GA, USA.
37. Hale GA, Kasow K, Madden R, et al. Mismatched family member donor transplantation for patients with refractory hematologic malignancies: Longterm follow-up of a prospective clinical trial. 48th Annual Meeting of the American Society of Hematology, 9-12 December 2006, Orlando, FL, USA.

38. Chen X, Hale GA, Barfield R, et al. Rapid immune reconstitution after a reduced-intensity conditioning regimen and a CD3-depleted haploidentical stem cell graft for paediatric refractory haematological malignancies. Br J Haematol 2006;135:524-32.

39. Lang P, Mueller I, Greil J, et al. Retransplantation with stem cells from mismatched related donors after graft rejection in pediatric patients. Blood Cells Mol Dis 2008;40:33-9.

40. Pfeiffer MM, Feuchtinger T, Teltschik HM, et al. Reconstitution of natural killer cell receptors influences natural killer activity and relapse rate after haploidentical transplantation of T- and B-cell depleted grafts in children. Haematologica 2010;95:1381-8.

41. Dufort G, Pisano S, Incoronato A, et al. Feasibility and outcome of haploidentical SCT in pediatric high-risk hematologic malignancies and Fanconi anemia in Uruguay. Bone Marrow Transplant 2011; e-pub ahead of print 18 July 2011.

42. Bonneville M, O’Brien RL, Born WK. Gammadelta T cell effector functions: a blend of innate programming and acquired plasticity. Nat Rev Immunol 2010;10:467-78.

43. Chiplunkar S, Dhar S, Wesch D, Kabelitz D. gammadelta T cells in cancer immunotherapy: current status and future prospects. Immunotherapy 2009;1:663-78.

44. Godder KT, Henslee-Downey PJ, Mehta J, et al. Long term disease-free survival in acute leukemia patients recovering with increased gammadelta $\mathrm{T}$ cells after partially mismatched related donor bone marrow transplantation. Bone Marrow Transplant 2007;39:751-7.

45. Huang XJ, Liu DH, Liu KY, et al. Haploidentical hematopoietic stem cell transplantation without in vitro $\mathrm{T}$-cell depletion for the treatment of hematological malignancies. Bone Marrow Transplant 2006;38:291-7.

46. Lu DP, Dong L, Wu T, et al. Conditioning including antithymocyte globulin followed by unmanipulated HLA-mismatched/haploidentical blood and marrow transplantation can achieve comparable outcomes with HLAidentical sibling transplantation. Blood 2006;107:3065-73.

47. Luo XH, Chang YJ, Xu LP, Liu DH, Liu KY, Huang XJ. The impact of graft composition on clinical outcomes in unmanipulated HLA-mismatched/ haploidentical hematopoietic SCT. Bone Marrow Transplant 2009;43: 29-36.

48. Xu LP, Liu KY, Liu DH, et al. The inferiority of G-PB to rhG-CSF-mobilized blood and marrow grafts as a stem cell source in patients with high-risk acute leukemia who underwent unmanipulated HLA-mismatched/haploidentical transplantation: a comparative analysis. Bone Marrow Transplant 2010;45:985-92.

49. Wang HX, Yan HM, Duan LN, et al. Haploidentical hematopoietic stem cell transplantation in child hematologic malignancies with G-CSF-mobilized marrow grafts without T-cell depletion: a single-center report of 45 cases. Pediatr Hematol Oncol 2009;26:119-28.

50. Luznik L, Engstrom LW, Iannone R, Fuchs EJ. Posttransplantation cyclophosphamide facilitates engraftment of major histocompatibility complexidentical allogeneic marrow in mice conditioned with low-dose total body irradiation. Biol Blood Marrow Transplant 2002;8:131-8.

51. Luznik L, Fuchs EJ. High-dose, post-transplantation cyclophosphamide to promote graft-host tolerance after allogeneic hematopoietic stem cell transplantation. Immunol Res 2010;47:65-77.

52. Luznik L, O’Donnell PV, Symons HJ, et al. HLA-haploidentical bone marrow transplantation for hematologic malignancies using nonmyeloablative conditioning and high-dose, posttransplantation cyclophosphamide. Biol Blood Marrow Transplant 2008; 14:641-50.

53. Brunstein CG, Fuchs EJ, Carter SL, et al. Alternative donor transplantation: results of parallel phase II trials using HLA-mismatched related bone marrow or unrelated umbilical cord blood grafts. Blood 2011;118:282-8.

54. Velardi A, Ruggeri L, Mancusi A, Aversa F, Christiansen FT. Natural killer cell allorecognition of missing self in allogeneic hematopoietic transplantation: a tool for immunotherapy of leukemia. Curr Opin Immunol 2009;21:525-30. 
55. Leung W. Use of NK cell activity in cure by transplant. Br J Haematol 2011;10:2141.

56. Bottino C, Moretta L, Pende D, Vitale M, Moretta A. Learning how to discriminate between friends and enemies, a lesson from Natural Killer cells. Mol Immunol 2004;41:569-75.

57. Moretta A, Locatelli F, Moretta L. Human NK cells: from HLA class I-specific killer Ig-like receptors to the therapy of acute leukemias. Immunol Rev 2008;224:58-69.

58. Locatelli F, Pende D, Maccario R, Mingari MC, Moretta A, Moretta L. Haploidentical hemopoietic stem cell transplantation for the treatment of high-risk leukemias: how NK cells make the difference. Clin Immunol 2009;133:171-8.

59. Leung W, Iyengar R, Triplett B, et al. Comparison of killer Ig-like receptor genotyping and phenotyping for selection of allogeneic blood stem cell donors. J Immunol 2005;174:6540-5.

60. Pende D, Marcenaro S, Falco M, et al. Anti-leukemia activity of alloreactive NK cells in KIR ligand-mismatched haploidentical HSCT for pediatric patients: evaluation of the functional role of activating KIR and redefinition of inhibitory KIR specificity. Blood 2009;113:3119-29.

61. Stern M, de Angelis C, Urbani E, et al. Natural killer-cell KIR repertoire reconstitution after haploidentical SCT. Bone Marrow Transplant 2010;45:1607-10.

62. Lowe EJ, Turner V, Handgretinger R, et al. T-cell alloreactivity dominates natural killer cell alloreactivity in minimally T-cell-depleted HLAnon-identical paediatric bone marrow transplantation. Br J Haematol 2003;123:323-6.

63. Huang XJ, Zhao XY, Liu DH, Liu KY, Xu LP. Deleterious effects of KIR ligand incompatibility on clinical outcomes in haploidentical hematopoietic stem cell transplantation without in vitro T-cell depletion. Leukemia 2007;21:848-51.

64. Symons HJ, Leffell MS, Rossiter ND, Zahurak M, Jones RJ, Fuchs EJ. Improved survival with inhibitory killer immunoglobulin receptor (KIR) gene mismatches and KIR haplotype B donors after nonmyeloablative, HLA-haploidentical bone marrow transplantation. Biol Blood Marrow Transplant 2010;16:533-42.

65. Cooley S, Weisdorf DJ, Guethlein LA, et al. Donor selection for natural killer cell receptor genes leads to superior survival after unrelated transplantation for acute myelogenous leukemia. Blood 2010;116:2411-9.

66. Almalte Z, Samarani S, Iannello A, et al. Novel associations between activating killer-cell immunoglobulin-like receptor genes and childhood leukemia. Blood 2011;118:1323-8.

67. Seggewiss R, Einsele H. Immune reconstitution after allogeneic transplantation and expanding options for immunomodulation: an update. Blood 2010;115:3861-8.

68. Kang Y, Chao NJ, Aversa F. Unmanipulated or CD34 selected haplotype mismatched transplants. Curr Opin Hematol 2008;15:561-7.

69. Amrolia PJ, Muccioli-Casadei G, Huls H, et al. Adoptive immunotherapy with allodepleted donor T-cells improves immune reconstitution after haploidentical stem cell transplantation. Blood 2006;108:1797-808.

70. Mielke S, Nunes R, Rezvani K, et al. A clinical-scale selective allodepletion approach for the treatment of HLA-mismatched and matched donor-recipient pairs using expanded $\mathrm{T}$ lymphocytes as antigen-presenting cells and a TH9402-based photodepletion technique. Blood 2008;111: 4392-402.

71. Perruccio K, Tosti A, Burchielli E, et al. Transferring functional immune responses to pathogens after haploidentical hematopoietic transplantation. Blood 2005;106:4397-406.

72. Dodero A, Carniti C, Raganato A, et al. Haploidentical stem cell transplantation after a reduced-intensity conditioning regimen for the treatment of advanced hematologic malignancies: posttransplantation CD8-depleted donor lymphocyte infusions contribute to improve T-cell recovery. Blood 2009;113:4771-9.

73. Edinger M, Hoffmann P, Ermann J, et al. CD4+CD25+ regulatory T cells preserve graft-versus-tumor activity while inhibiting graft-versus-host disease after bone marrow transplantation. Nat Med 2003;9:1144-50.

74. Federmann B, Hägele M, Pfeiffer M, et al. Immune reconstitution after haploidentical hematopoietic cell transplantation: impact of reduced intensity conditioning and CD3/CD19 depleted grafts. Leukemia 2011;25:121-9.

75. Chen X, Barfield R, Benaim E, et al. Prediction of T-cell reconstitution by assessment of $\mathrm{T}$-cell receptor excision circle before allogeneic hematopoietic stem cell transplantation in pediatric patients. Blood 2005; 105:886-93.

76. Vago L, Perna SK, Zanussi M, et al. Loss of mismatched HLA in leukemia after stem-cell transplantation. N Engl J Med 2009;361:478-88.

77. Villalobos IB, Takahashi Y, Akatsuka Y, et al. Relapse of leukemia with loss of mismatched HLA resulting from uniparental disomy after haploidentical hematopoietic stem cell transplantation. Blood 2010;115:3158-61.

78. Lewalle P, Triffet A, Delforge A, et al. Donor lymphocyte infusions in adult haploidentical transplant: a dose finding study. Bone Marrow Transplant 2003;31:39-44.

79. Rizzieri DA, Storms R, Chen DF, et al. Natural killer cell-enriched donor lymphocyte infusions from A 3-6/6 HLA matched family member following nonmyeloablative allogeneic stem cell transplantation. Biol Blood Marrow Transplant 2010;16:1107-14.

80. Pfeiffer M, Lang P, Schumm M, et al. IL-15 activated CD3/19 depleted grafts for haploidentical transplantation in children: strongly increased NK activity in vitro and excellent tolerability in vivo. 34th Annual Meeting of the European Group for Blood and Marrow Transplantation, 30 March-2 April 2008, Florence, Italy.

81. Lang P, Barbin K, Feuchtinger T, et al. Chimeric CD19 antibody mediates cytotoxic activity against leukemic blasts with effector cells from pediatric patients who received T-cell-depleted allografts. Blood 2004;103:3982-5.

82. Bargou R, Leo E, Zugmaier G, et al. Tumor regression in cancer patients by very low doses of a T cell-engaging antibody. Science 2008;321:974-7.

83. Handgretinger R, Zugmaier G, Henze G, Kreyenberg H, Lang P, von Stackelberg A. Complete remission after blinatumomab-induced donor T-cell activation in three pediatric patients with post-transplant relapsed acute lymphoblastic leukemia. Leukemia 2011;25:181-4.

84. Kerst G, Kreyenberg H, Roth C, et al. Concurrent detection of minimal residual disease (MRD) in childhood acute lymphoblastic leukaemia by flow cytometry and real-time PCR. Br J Haematol 2005;128:774-82.

85. Leung W, Campana D, Yang J, et al. High success rate of hematopoietic cell transplantation regardless of donor source in children with very high-risk leukemia. Blood 2011;118:223-30. 